\title{
Discrimination between Gamma and Rayleigh Models
}

\author{
M. S. Ravikumar ${ }^{1, *}$, R. R. L. Kantam ${ }^{2}$ \\ ${ }^{1}$ Department of Community Medicine \\ Konaseema Institute of Medical Sciences \& Research Foundation/ General Hospital \\ Amalapuram-533201, Andhra Pradesh, India. E-mail: ${ }^{1}$ msrk.raama@ gmail.com \\ ${ }^{2}$ Department of Statistics, Acharya Nagarjuna University \\ Nagarjunanagar-522510, Andhra Pradesh, India. E-mail: ${ }^{2}$ kantam.rrl@gmail.com \\ ${ }^{*}$ Corresponding author
}

(Received June 1, 2016; Accepted July 2, 2016)

\begin{abstract}
The two popular life testing models are considered to verify whether one can be an alternative to other. The motivation for this study is as follows. It is well known that the cumulative distribution function of Rayleigh distribution can be analytically inverted where as it is not so with Gamma distribution. Generally analytical inversion of cumulative distribution function would be advantageous in the study of problems of inference. "Whether this advantage can be explored in assessing the discrimination or otherwise of the two models" is studied in this paper.
\end{abstract}

Keywords: Rayleigh distribution, Gamma distribution, likelihood ratio criterion.

\section{Introduction}

The cumulative distribution function and probability density function of Gamma distribution with shape parameter 2 and Rayleigh distribution (Weibull with shape parameter 2) are given by Eqs. $(1-4)$ :

$F_{0}(x)=1-e^{-(x / \sigma)}\left(1+\frac{x}{\sigma}\right) ; x \geq 0, \sigma>0$.

and

$f_{0}(x)=\frac{x}{\sigma^{2}} e^{-(x / \sigma)} ; x \geq 0, \sigma>0$.

$F_{1}(x)=1-e^{\left(-x^{2} /\left(2 \sigma^{2}\right)\right)} ; 0 \leq x<\infty, \sigma>0$.

and

$f_{1}(x)=\frac{x}{\sigma^{2}} e^{-\left(x^{2} / 2 \sigma^{2}\right)} ; 0 \leq x<\infty, \sigma>0$.

Here $\sigma$ is called the scale parameter in the above models. We see in most of the text books that the frequency curves of the two distributions look alike. The cumulative distribution function of Rayleigh distribution is analytically invertible whereas that of Gamma distribution is an incomplete Gamma function extensively tabulated. This advantage of Rayleigh distribution is the motivation to study whether it can be an alternative to Gamma with shape parameter 2. Some of the authors working in this direction are of Gupta and Kundu (2003) for the Weibull and the 
generalised exponential distributions, Gupta and Kundu (2004) for the Gamma and generalised exponential distributions, Kundu and Manglick (2004) for the Weibull and log-normal distributions, Kundu (2005) for normal and Laplace distributions, Kundu et al. (2005) for the lognormal and the generalised exponential distributions, Kundu and Manglick (2005) for the Gamma and log-normal distributions, Kundu and Raqab (2007) for the log-normal and generalised Rayleigh distributions, Dey and Kundu (2009) for log-normal, Weibull and generalised exponential distributions, Dey and Kundu (2010) for the log-normal and log-logistic distributions, Dey and Kundu (2012a) for the Weibull and log-normal distributions for type - II censored data, Dey and Kundu (2012b) for bivariate generalised exponential and bivariate Weibull distributions, Rao and Kantam (2013) for generalised exponential distribution and some life test models based on population quantiles, Rao et al. (2013) for half normal and half logistic distributions, Rao and Kantam (2014) for log-logistic and Rayleigh distributions, Kantam et al. (2014a) for likelihood ratio test for linear failure rate and exponential distributions, Kantam et al. (2014b) for linear failure rate and Rayleigh distributions, Prasad and Kantam (2015) for the test procedure between probability models and Kantam and Ravikumar (2015) for Burr type XII and Weibull-exponential distributions. We made an attempt in that direction through likelihood ratio criterion.

The likelihood ratio criterion is narrated in Section-2. The adopted simulation study results and conclusions are given in Section-3 and Section-4.

\section{Likelihood Ratio Criterion}

In our study Gamma 2 is taken as null population and Rayleigh is taken as an alternative population.

i.e., $H_{0}$ : A given sample $\left(x_{1}, x_{2}, \ldots, x_{n}\right)$ belongs to Gamma 2 model.

$H_{1}$ : The sample $\left(x_{1}, x_{2}, \ldots, x_{n}\right)$ belongs to Rayleigh model.

The MLE of $\sigma$ of Gamma 2 is known as (Eq. (5))

$$
\frac{\partial \log L_{0}}{\partial \sigma}=0 \Rightarrow \hat{\sigma}=\frac{\bar{x}}{2}
$$

Similarly MLE of $\sigma$ of Rayleigh is known to be (Eq. (6))

$$
\frac{\partial \log L_{1}}{\partial \sigma}=0 \Rightarrow \hat{\sigma}=\sqrt{\frac{\sum_{i=1}^{n} x_{i}^{2}}{2 n}}
$$

The LR statistic is defined as $\lambda=\ln \left(\frac{L_{1}}{L_{0}}\right)$ evaluated at the respective MLEs of $\sigma$.

i.e., $\lambda=2 n \ln (\bar{x})-n \ln \left(\sum_{i=1}^{n} x_{i}^{2}\right)+n \ln (n)+n-n \ln (2)$. 
International Journal of Mathematical, Engineering and Management Sciences

Vol. 1, No. 2, 89-92, 2016

https://dx.doi.org/10.33889/IJMEMS.2016.1.2-010

If $\lambda$ is negative then the null hypothesis that the sample is from Gamma is accepted. In other words for a sample that is generated from Gamma, a negative value of $\lambda$ yields correct decision otherwise it will give wrong decision.

Therefore $\lambda$ can be used as a criterion for testing the above $H_{0}$ vs. $H_{1}$. Hence the percentiles of $\lambda$ are essential for this purpose. However we adopted a different simulation study to get the proportion of correct decision vs. a wrong decision as given in Section 3.

\section{Simulation Study}

Samples of size 5, 10, 15, 20 and 25 are generated from Gamma 2 and at those samples the $\sigma$ of Gamma 2 as well as Rayleigh distribution is estimated by ML method. The respective likelihood and the LR criterion $\lambda$ are evaluated. The proportion of negative and positive $\lambda$ 's are computed out of the used simulation runs. The details are given in the following Table 1.

\begin{tabular}{|c|c|c|}
\hline Sample Size $(\boldsymbol{n})$ & $\begin{array}{c}\text { Number of times } \\
\boldsymbol{\lambda} \text { is negative }\end{array}$ & $\begin{array}{c}\text { Number of times } \\
\boldsymbol{\lambda} \text { is positive }\end{array}$ \\
\hline 5 & 0.38 & 0.62 \\
\hline 10 & 0.57 & 0.43 \\
\hline 15 & 0.63 & 0.37 \\
\hline 20 & 0.69 & 0.31 \\
\hline 25 & 0.75 & 0.25 \\
\hline
\end{tabular}

Table 1. Proportion of negative and positive $\lambda$ 's

These results reveal that when sample size is more than 15 Rayleigh cannot be an alternative to Gamma 2. In small samples that are of size 5, 10 Rayleigh can be used as a reasonable alternative to Gamma 2. Specifically over increased simulation runs for sample sizes 5, 10 we observe the following Table 2 .

\begin{tabular}{|c|c|c|}
\hline Sample Size $(\boldsymbol{n})$ & Count of Negative $\boldsymbol{\lambda}$ & Count of Positive $\boldsymbol{\lambda}$ \\
\hline 5 & 0.59489 & 0.40511 \\
\hline 10 & 0.43973 & 0.56027 \\
\hline
\end{tabular}

Table 2. Increased simulation runs for sample sizes 5 and 10

This further shows a fifty-fifty choice of Gamma 2 vs. Rayleigh up to a sample of size 10.

\section{Conclusion}

We therefore conclude that in samples of size as small as up to 10. Gamma 2 and Rayleigh are equally likely models for a sample generated from either of the two. For other large samples they make a distinction.

\section{References}

Dey, A. K., \& Kundu, D. (2009). Discriminating among the log-normal, Weibull, and generalized exponential distributions. IEEE Transactions on Reliability, 58(3), 416-424. 
International Journal of Mathematical, Engineering and Management Sciences

Vol. 1, No. 2, 89-92, 2016

https://dx.doi.org/10.33889/IJMEMS.2016.1.2-010

Dey, A. K., \& Kundu, D. (2010). Discriminating between the log-normal and log-logistic distributions. Communications in Statistics-Theory and Methods, 39(2), 280-292.

Dey, A. K., \& Kundu, D. (2012a). Discriminating between the Weibull and log-normal distributions for Type-II censored data. Statistics, 46(2), 197-214.

Dey, A. K., \& Kundu, D. (2012b). Discriminating between bivariate generalized exponential and bivariate Weibull distributions. Chilean Journal of Statistics, 3(1), 93-110.

Gupta, R. D., \& Kundu, D. (2003). Discriminating between Weibull and generalized exponential distributions. Computational Statistics \& Data Analysis, 43(2), 179-196.

Gupta, R. D., \& Kundu, D. (2004). Discriminating between Gamma and generalized exponential distributions. Journal of Statistical Computation \& Simulation, 74(2), 107-121.

Kantam, R. R. L. \& Ravikumar, M. S. (2015). Discrimination between Burr Type XII distribution and Weibull-exponential distribution. Conference Proceedings of National Seminar on Recent Developments in Applied Statistics - Golden Research Thoughts, 1-11.

Kantam, R. R. L., Priya, M. C., \& Ravikumar, M. S. (2014a). Likelihood ratio type test for linear failure rate distribution vs. exponential distribution. Journal of Modern Applied Statistical Methods, 13(1), 174-186.

Kantam, R. R. L., Priya, M. Ch. \& Ravikumar, M. S. (2014b). Discrimination between linear failure rate distribution and Rayleigh distribution. Journal of Reliability and Statistical Studies, 7, Special Issue, 917.

Kundu, D. (2005). Discriminating between normal and Laplace distributions. In Advances in Ranking and Selection, Multiple Comparisons, and Reliability (pp. 65-79). Birkhäuser Boston.

Kundu, D., \& Manglick, A. (2004). Discriminating between the Weibull and log-normal distributions. Naval Research Logistics, 51(6), 893-905.

Kundu, D., \& Manglick, A. (2005). Discriminating between the log-normal and Gamma distributions. Journal of the Applied Statistical Sciences, 14, 175-187.

Kundu, D., \& Raqab, M. Z. (2007). Discriminating between the generalized Rayleigh and log-normal distribution. Statistics, 41(6), 505-515.

Kundu, D., Gupta, R. D., \& Manglick, A. (2005). Discriminating between the log-normal and generalized exponential distributions. Journal of Statistical Planning and Inference, 127(1), 213-227.

Prasad, G. and Kantam, R. R. L. (2015). A test procedure to discriminate between probability models. International Journal of Scientific and Innovative Mathematical Research, 3(11), 54-58.

Rao, B. S., \& Kantam, R. R. (2013). Discriminating between generalized exponential distribution and some life test models based on population quantiles. Journal of Modern Applied Statistical Methods, 12(2), 336-343.

Rao, B. S., \& Kantam, R. R. L. (2014). Discriminating Between Log-Logistic and Rayleigh Distributions. Pakistan Journal of Statistics and Operation Research, 10(1), 1-7.

Rao, B. S., Kumar, C. H. S., Kantam, R. R. L. \& Rosaiah, K. (2013). Discriminating between half normal and half logistic distributions. International Journal of Computer Information Systems, 7(4), 59-63. 